\title{
EL SINTAGMA DETERMINANTE EN EDUCACIÓN
} SECUNDARIA. PROPUESTA DE ACTUALIZACIÓN METODOLÓGICA EN LA ENSEÑANZA DE LA SINTAXIS

\author{
Mario Casado Mancebo ${ }^{1}$ \\ Universidad Nacional de Educación a Distancia
}

Recibido 11/06/2020 Aceptado 16/11/2020

Las asignaturas de Lengua castellana y literatura del plan de es-
tudios de Educación secundaria están marcadas por un enfoque
tradicional que impide visualizar toda una serie de problemas
teóricos que la lingüística superó en las últimas décadas del siglo
XX. El conservadurismo gramatical en la enseñanza de español
L1 impide que la educación pueda caminar en paralelo con los
avances en la ciencia lingüística. En este trabajo se ofrece una
propuesta de actualización metodológica centrada en el problema
del tratamiento de las estructuras nominales en la Educación se-
cundaria. Junto a la propuesta se ofrece un marco teórico y una
amplia revisión bibliográfica crítica del tratamiento de los deter-
minantes en los materiales de didáctica del español que eviden-
cian el problema metodológico y justifican la propuesta didáctica.

$\exists$ Subjects of Lengua castellana y Literatura in the Secondary Education curriculum are determined by a traditional approach that keeps us from spotting a bunch of theoretical problems overcome by linguistics through last decades of XXth century. This conservatism at grammar teaching prevents education from advancing together with linguistic progresses. In this paper, there is a proposal for a methodological update focused on the treatment of nominal phrases in Secondary Education. Together with it, you will find a theoretical framework and an extensive bibliographical review of Spanish language teaching resources which evidence the problem and justify the teaching proposal.

DOI

https://doi.org/10.15366/didacticas2020.23.004

PALABRAS ClaVE

Educación Secundaria Obligatoria; Bachillerato; Didáctica de la Lengua; sintagma nominal; sintagma determinante.

Secondary Education; Bachillerato; Spanish language teaching; noun phrase; determiner phrase. 


\section{INTRODUCCIÓN}

Lengua castellana y literatura es una de las asignaturas centrales del plan de estudios de Educación Secundaria Obligatoria. Su currículo incluye objetivos tan fundamentales como comprender y producir texto o comprender los mecanismos de la lengua y saber aplicarlos a la hora de manejar textos de forma autónoma. Estos complejos objetivos en muchas ocasiones se ven dificultados por el enfoque que se elige para desarrollar los contenidos prescritos. Un enfoque marcadamente tradicional que obvia muchos problemas inherentes a la teoría y, lo que es peor, las soluciones que la lingüística ha dado en la última parte del siglo XX. La mayoría de los libros de texto oficiales y los manuales de didáctica de la lengua no ofrecen una solución a este problema, puesto que los unos se alimentan de los contenidos de los otros, impidiendo el avance de la enseñanza de la lengua de forma paralela a los avances en los estudios de lingüística.

Uno de los problemas clave de los contenidos en el ámbito de la competencia gramatical es el de las estructuras nominales. Este trabajo constituye una propuesta didáctica para la implementación del sintagma determinante en la asignatura de Lengua castellana en ESO y Bachillerato. Se parte del epígrafe del sintagma o grupo nominal del currículo oficial para, desde ahí, ampliar de forma coherente los tipos de estructuras nominales de la lengua. Y, puesto que este tema es uno de los principales del currículo de esta asignatura y está presente en todos los cursos, no se ha precisado un curso concreto para la aplicación de la propuesta. Los contenidos expuestos son susceptibles de ser adaptados fácilmente al nivel que corresponda. De hecho, en varias secciones, se ofrecen sugerencias de adaptación y nociones complementarias.

El trabajo se estructura en dos bloques principales: el primero, un marco teórico (apartado 2) que contiene todos los conceptos fundamentales que el docente debe conocer para poder comprender la importancia de la propuesta didáctica. Se exponen de forma argumentada los diferentes motivos por los que, alrededor de los años 80, se evidenció la necesidad de contemplar un segundo tipo de estructuras nominales. El segundo, compuesto de los apartados 3 y 4, la revisión del tratamiento de los determinantes en Educación secundaria para evidenciar el problema al que nos enfrentamos y la propuesta didáctica y metodológica para corregirlo. Estos dos apartados no deben confundirse: el marco teórico no contiene de ninguna manera una explicación apta para llevar al aula de Educación secundaria. Como ya se ha mencionado, está pensado como base conceptual para el docente; por ello, en él se manejan conceptos gramaticales que un especialista puede comprender, pero que pueden no ser accesibles a los estudiantes. El contenido destinado a los alumnos se ofrece en el apartado 4, junto a una serie de orientaciones metodológicas. El trabajo se cierra en el apartado 5 con unas breves consideraciones finales al margen de la propuesta. 


\section{MARCO TEÓRICO}

\subsection{Los determinantes en la tradición lingüística española}

Tradicionalmente, como es habitual encontrar en los materiales didácticos de lengua, los sintagmas de tipo nominal se consideraban como la expansión de un nombre (núcleo) y sus dependencias optativas: a la derecha, complementos adjetivales o preposicionales y, a la izquierda, especificadores gramaticales (artículos, adjetivos determinativos...). El eje vertebrador de las estructuras nominales era el nombre y el determinante no era más que un complemento periférico, como se puede corroborar en el tratamiento gramatical que recibía: sin una función sintáctica clara y sin un paradigma gramatical específico. Como señalan Bosque y Gutiérrez-Rexach (2009:105), las gramáticas tradicionales no solían si quiera incluir una categoría gramatical específica para referirse a las palabras que, de forma constante, aparecían en los especificadores de los sintagmas nominales (SSNN). En la gramática de Andrés Bello (1847), de las más antiguas que se usan como referencia para el análisis historiográfico de los determinantes, se incluye una sección dedicada a lo que hoy denominaríamos clases de palabras (Bello, 2002: cap. 2); sin embargo, las únicas clases tratadas en él son el sustantivo, el adjetivo, el verbo, el adverbio, la preposición, la conjunción y la interjección. Sí se incluyen, sin embargo, varias secciones referidas a la definición y uso del artículo (Bello, 2002: cap. 14 y 31).

La gramática de la Real Academia de 1931 introduce en su estudio de la analogía ("la parte de la Gramática que enseña el valor de las palabras consideradas aisladamente", RAE, 1931: §1.1) un apartado para el artículo como categoría distinta. De la estructuración del estudio, se desprende su consideración paralela a las otras clases de palabras (en esta obra, nombre substantivo, nombre adjetivo, pronombre, verbo, etc.).

En la gramática de la Academia, al igual que en la de Bello, no se encuentra ningún punto de contacto sintáctico entre artículos y nombres. Si bien, en el caso de los adjetivos, estas gramáticas sí especifican la relación semántica que existe entre ambos (en la gramática de la RAE, concretamente, se habla ya de complementos del nombre, 1931: $\S 14)$, en el caso de los artículos simplemente encontramos, en el apartado correspondiente, una descripción de los valores semánticos referenciales que estos aportan en los nombres y adjetivos sin considerarlos estructuralmente relacionados. Muy probablemente, el motivo de esta ausencia esté en la consideración que la gramática tradicional otorgaba a la clase que hoy conocemos como determinantes. Como explica la RAE, los pronombres demostrativos "pierden el acento que llevan (...) y se convierten en adjetivos determinativos cuando van unidos al nombre"1 (1931: 36).

Entendemos, entonces, que en aquel momento no se apreciaba la diferencia funcional que hoy reconocemos entre los complementos del nombre - esos "adjetivos" - y los especificadores de este - los determinantes-. Dejando a un lado el hecho evidente de que determinantes y adjetivos son paradigmas diferentes (el primero es un repertorio de ele-

1 Añado la cursiva como llamada de atención. 
mentos funcionales cerrado y el segundo es un repertorio de elementos léxicos abierto), existe una diferencia funcional específica que separa la clase de los determinantes de la de los adjetivos. Como explica Fernández Leborans (2003: 33) el complemento de un núcleo - la posición derecha o interna- aumenta el contenido descriptivo de este; es decir, tomando el ejemplo de la autora, en el libro de matemáticas, el sintagma de matemáticas cumple la función de añadir contenido descriptivo ${ }^{2}$. Frente a esto, el artículo el no aporta ninguna noción descriptiva extra, sino que proporciona la referencia; es decir, añade al grupo libro de matemáticas la función gramatical que permite interpretar que estamos refiriéndonos a un libro específico que ha sido previamente mencionado. De este modo, en términos semánticos, los complementos del nombre aportan datos relativos a la intensión de este, y los determinantes aportan datos relativos a la extensión ${ }^{3}$.

En la tradición estructuralista española tampoco era común encontrar la referencia a los determinantes. Alarcos Llorach (1951: §53) no llega a referirse en ningún momento a una clase gramatical específica para los determinantes; sin embargo, si que nota, por primera vez, que tienen una función gramatical específica al mismo nivel que otros exponentes - como él denomina a los ítems funcionales ${ }^{4}$ - como el caso, el género y el número. Si bien es cierto que únicamente reconoce esta función en los artículos, dejando aún como "adjetivos" a los posesivos y los demostrativos, el que atribuya a los artículos en su clasificación la categoría de morfemas ('exponentes dentro del plano de las unidades lingüísticas con significado', Alarcos Llorach 1951: §30) es un avance muy importante, puesto que abre el camino hacia un enfoque más adecuado desde el punto de vista científico-teórico.

De igual forma, en la propuesta de Alarcos Llorach, se aprecia un primer acercamiento a las consecuencias sintácticas de los determinantes. En las otras gramáticas que hemos mencionado, la única significación que se reconocía a los artículos era el mero incremento referencial dentro de la semántica del nombre. Alarcos Llorach (1951: §53) reconoce que "el artículo que lleve éste [el sujeto de un predicado] es regido por el predicado" y que "éste exige el artículo" . Como en el caso anterior, haber llegado a la rección de los sujetos y al hecho de que la presencia constante de un artículo en ellos no es una casualidad ni un accidente de la gramática resulta muy prometedor, ya que lo primero empieza a romper con la tradicional concepción categórica del sujeto por un lado y el predicado por otro, al mismo nivel, sin relación posible entre ambos: si los predicados "rigen" a sus sujetos, debe existir alguna relación sintáctica entre ambos; y lo segundo abre una vía hacia un estudio de la función de los determinantes en el sintagma nominal (SN): si su presencia no es optativa en un tipo de SSNN (los que tienen función de sujeto), alguna

2 Utilizo el concepto descriptivo como opuesto a funcional o gramatical; es decir, es contenido descriptivo aquel que corresponde a nociones del mundo y no a funciones y conceptos de la gramática.

3 Para los conceptos de intensión y extensión, véase, por ejemplo, Escandell Vidal (2004).

4 Al contrario de lo que observábamos en la nota 2 los ítems o categorías funcionales son aquellos con contenido o significado funcional, no designativo (Bosque y Gutiérrez-Rexach, 2009: 109).

5 Añado la cursiva como llamada de atención. 
regla debe operar sobre ellos para diferenciarlos de aquellos en los que el determinante no es obligatorio (los SSNN con función de complemento directo, por ejemplo: Juan compra calabazas).

En el primer modelo teórico de la gramática generativa, aún se utilizaba el SN como fórmula generalizada. De hecho, la regla de derivación de SSNN en la Teoría estándar era la conocida $S N \rightarrow($ Det $)+N($ Eguren y Fernández Soriano, 2004: 77). En primer lugar, debemos notar que, por primera vez, se formaliza una posición específica $-\mathrm{y}$ con un nombre de paradigma: determinantes (Det)- para los elementos que ocupan la posición izquierda de los SSNN. Aparece la categoría del determinante, que en este primer momento no es más que un adjunto paralelo al complemento de tipo "calificativo" que puede aparecer al margen derecho del SN, como se desprende de propuestas como la de Fernández Leborans (1983), que propone el esquema $S N=[\operatorname{Adjunto(s)I]}+\operatorname{Núcleo}+[\operatorname{Adjunto(s)}$ II] (p. 60). Es importante, sin embargo, destacar como la autora recurre a los números romanos para establecer claramente dos categorías de adjuntos del nombre: los de tipo I, que especifica como "determinante(s)", y los de tipo II, "adjetivos(s)" ${ }^{6}$, distinguiendo así, como veíamos más arriba, entre dos paradigmas claramente diferenciados: el de los determinantes y el de los complementos del nombre. Que en estas propuestas el determinante se represente como optativo (entre paréntesis, corchetes, denominándolo adjunto, etc.) nos sirve de testimonio de la concepción nominocéntrica que aún existía de los sintagmas de tipo nominal. No obstante, a partir de este momento, la categoría gramatical de los determinantes se generalizará junto a todas las demás, uniéndose al grupo de las categorías funcionales.

\subsection{Sintagma nominal y sintagma determinante}

Si bien veíamos que la categoría de los determinantes como clase discreta emerge de forma estable en las teorías generativas, hay que esperar hasta el modelo de Principios y $\mathrm{Pa}$ rámetros, en los años 80, para que, al hilo de la propuesta de Abney (1987), se reconsideren las formas de categorizar los SSNN. Un cambio fundamental en este nuevo modelo fue la introducción de la Condición de Ramificación Binaria ${ }^{7}$ que, como recogen Eguren y Fernández Soriano, se da en los años 80 (2004: nota 35) como forma de adecuar metodológicamente la formalización de estructuras. Un sintagma binario permite explicar de forma óptima las interpretaciones que se generan de las ambigüedades estructurales y, además, es el análisis con mayor univocidad y a la vez más informativo (Brucart y Hernanz, 2015:37).

6 Fernández Leborans no olvida, evidentemente, la posibilidad de complementación preposicional del nombre. Como aclara en la nota 5 de su artículo (Fernández Leborans, 1983), al nombrar a los adjuntos de tipo II "adjetivo(s)", se re fiere a "adjetivos funcionales". Lo mismo que yo he denominado unas líneas más arriba "complementos de tipo "calificativo"'.

7 La ramificación binaria exige que los sintagmas se ramifiquen estrictamente de dos en dos siguiendo el esquema "núcleo + complemento". Esto unifica el análisis de todos los sintagmas. Es una estructura que forma parte de la gramática universal de las lenguas naturales. 
La condición universal de binarismo supuso un primer problema en los análisis del $\mathrm{SN}$, que, como hemos visto, solían ramificarse en tres para incluir los dos tipos de complemento (adjunto(s) I y II, cap. 2.1). La teoría X-con-barra (Chomsky, 1970 y Jackendoff, 1977, como recogen Bosque y Gutiérrez-Rexach, 2009), supuso una solución. Esta propuesta amplía la idea del binarismo proponiendo la siguiente estructura universal de sintagmas:

1)<smiles>[Y]C([Al])C=[V]</smiles>

X representa el núcleo del sintagma en cuestión (SX), SY es el complemento del núcleo que, junto a este, se proyecta de forma binaria, como exige la Condición de Ramificación Binaria, en X' ('X-con-barra'). A esta primera proyección (X') se puede añadir otro complemento, el especificador (SZ). Un especificador tiene dos características fundamentales: (1) es un sintagma, no un núcleo, y (2) complementa a una proyección X-conbarra, y no directamente a un núcleo ${ }^{8}$. Esto significa que $\mathrm{SZ}$ en el diagrama de 1 complementa a X + SZ, no solo a X.

La estructura X-con-barra permitía formalizar los SSNN con determinante de forma binaria, puesto que contempla la posición de especificador del núcleo: una posición de complemento a la izquierda, exactamente lo que parecían los determinantes. Sin embargo, como primer inconveniente, tenemos la característica (1) de los especificadores: los elementos que ocupen la posición de especificador deben ser sintagmas, no categorías mínimas. Un segundo inconveniente surgiría con las estructuras nominales con adjetivos ocupando el especificador (p. ej., antiguos libros de historia). Como hemos visto, el especificador complementa al cómputo formado por núcleo y complemento, lo que encaja con el ejemplo propuesto: el sintagma adjetival antiguos no se refiere por separado a libros sino que se trata de libros de historia que son antiguos. Entonces, su estructura sería la que se reproduce en (2). Un SN como este no tiene un lugar disponible para el determinante, puesto que el especificador del núcleo está ocupado por el sintagma antiguos.

2) $[\mathrm{sN}[\mathrm{sA}$ antiguos] libros [sp de historia $]]$

Estos argumentos de corte puramente estructural se añaden a aquello que ya notó Alarcos Llorach (1951): el determinante es obligatorio en los sujetos y seleccionado por los predicados ( ${ }^{*}$ Niño está llorando vs. El niño está llorando). Además, las reglas de se-

8 Principio de Uniformidad estructural (Bosque y Gutiérrez-Rexach, 2009, p. 207): las categorías mínimas o núcleos solo pueden ocupar la posición X del esquema X-con-barra; los sintagmas o expresiones máximas solo pueden ocupar la posición de complemento (SY) o la de especificador (SZ). 
lección sintáctica que hacen posible la aparición de determinantes crean secuencias con significado diferente:

3) a. Como galletas.

b. Me como las galletas.

En el segundo caso (3b), como notaban las gramáticas tradicionales, el hablante se refiere a unas galletas específicas que el interlocutor conoce previamente. Esto es: no solo hay posiciones donde la sintaxis exige la presencia de un determinante, sino que, en aquellas en las que su presencia parece optativa (como en 3, en realidad no lo es. La omisión del determinante o su presencia crea secuencias con significado diferente. Si ya habíamos concluido que los determinantes son visibles a la sintaxis, el hecho de que su presencia altere los significados los lleva más allá: los hace visibles al componente semántico-interpretativo. En esta línea, resulta muy clara la importancia de la capacidad referencial de los SSNN a la hora de distinguir su funcionalidad en la oración. Los SSNN con determinante son habitualmente referenciales; es decir, tienen poder semántico para referir a entidades concretas, mientras que los SSNN suelen ser predicativos o intensionales (Fernández Leborans, 2003: 39) -aportan lecturas conceptuales o de clase-.

Todas estas razones: (i) que los determinantes sean una categoría gramatical como las demás, (ii) que sobre ellos existan rasgos de selección sintáctica y, (iii) que esa selección produzca secuencias significativamente diferentes en torno a la referencia, son las que llevaron a concluir que existen dos tipos de estructuras nominales diferenciadas: por un lado, los SSNN, aquellas estructuras que no cuentan con determinante y que tienen unas posiciones específicas que, cuando se manipulan, producen la alteración de los significados; y, por otro, los sintagmas determinantes (SSDD), secuencias cuyo núcleo es el propio determinante, puesto que es visible a las operaciones sintácticas y de significado.

Hay que añadir, además, que el considerar a los determinantes núcleos de un sintagma de su misma categoría gramatical también va a favor de los principales axiomas de la gramática: por un lado, de la condición de endocentricidad de la gramática, que establece que los sintagmas de las lenguas se deben identificar y legitimar sintácticamente a través de sus núcleos. Es decir: todo sintagma es una proyección de un núcleo de su mismo signo categorial. Y, por otro, de la condición de maximidad, que establece que todo núcleo o categoría mínima debe proyectarse como sintagma o expresión máxima. Como veíamos anteriormente, el tener a la categoría de los determinantes dentro del SN como categoría mínima junto al nombre núcleo resultaba contradictorio a los principios del lenguaje (Principio de Uniformidad estructural, nota 9), puesto que, en la práctica se trata de dos categorías mínimas - dos posibles núcleos- compitiendo por ser el núcleo del sintagma.

De este modo, sabiendo ya que las estructuras nominales pueden ser de dos tipos, queda por aclarar cómo se estructuran sintácticamente. El hecho de contar con una pro- 
yección máxima con el signo determinante podría salvar la hipótesis de los determinantes en el especificador del SN; sin embargo, hemos de recordar que la posición de especificador queda bajo el dominio de la proyección máxima del núcleo. Es decir: un sintagma determinante (SD) en el especificador de un SN está bajo el dominio del nombre. Queda dentro del dominio sintáctico del SN. Como ya hemos visto en varias ocasiones, el SD debe ser visible a la sintaxis y sus reglas. Si este queda camuflado dentro de la estructura del SN, no hay forma de que la sintaxis pueda acceder a él o "visualizarlo" para generar las reglas de distribución vistas.

Para finalizar, es importante puntualizar el lugar en el que queda una de las categorías tradicionales dentro del nuevo sistema de estructuras nominales. Ahora que hemos reconocido la importancia del determinante, por un lado, y de la referencia del grupo nominal en cuestión por otro, se hace evidente la estrecha relación entre el SD y la categoría del pronombre, como señala Bosque (2007: cap.9). Los pronombres, efectivamente, son conocidos desde la gramatical tradicional por su poder referencial: "Pronombre es la parte de la oración que designa una persona o cosa sin nombrarla” (RAE, 1931: §69), y, en este sentido, parece que comparten ciertos usos funcionales con los determinantes. La gramática tradicional, de hecho, notó algunos de estos usos compartidos, utilizando las etiquetas de pronombre demostrativo, pronombre posesivo, etc. opuestas a los usos de estas formas como determinantes (adjetivos demostrativos, adjetivos posesivos, etc.). Como explica Fernández Leborans (2003, §3.2.2-3.2.4), la distinción entre determinantes y pronombres parece «pro-intuitiva», puesto que permite diferenciar una serie de expresiones que se combinan con nombres - los determinantes- de otras que conforman expresiones máximas (o sintagmas) por sí mismas; sin embargo, es frecuente encontrar definiciones en las que, en lugar de aludir al pronombre por su capacidad referencial, se dice que su función es sustituir al nombre y que, por tanto, forman SSNN. Esto no es cierto, puesto que no pueden aparecer en el lugar de los SSNN, sino en el de los SSDD ${ }^{9}$, como señalan tanto Fernández Leborans (2003: 42) como Eguren y Fernández Soriano (2006: 24). Si bien de cara a las categorías gramaticales puede resultar interesante mantener la distinción entre los determinantes, que aportan referencia, y los pronombres, que la poseen intrínsecamente, de cara a la sintaxis las dos comparten el mismo tipo de estructura: el $\mathrm{SD}^{10}$. Como hemos visto, esta estructura es la única con poder de recuperar referencias del discurso. Si mantuviésemos que los pronombres se proyectan en forma de SN, estaríamos obviando su capacidad para referir a otros SSDD del discurso.

9 En las estructuras donde aparece un determinante no puede aparecer un pronombre y viceversa:

- Quiero [so las galletas de chocolate].

- $\quad$ [so Las] quiero.

○ *[so Las [sN las]] quiero.

10 Esta solución no es inusual. Pensemos que es exactamente lo que sucede con las tradicionales categorías de artículo, demostrativo, posesivo, etc. Aunque se mantienen como elemento gramatical con paradigmas propios, todas ellas han quedado supeditadas a la categoría de determinante, como observaremos más adelante. 


\section{LOS DETERMINANTES EN EDUCACIÓN SECUNDARIA}

Antes de pasar al desarrollo de los contenidos didácticos, considero necesario revisar brevemente el tratamiento que se les da a los determinantes en la enseñanza media como forma de justificar la propuesta. Como observaremos a continuación, no es un tratamiento ni adecuado ni coherente con las reglas de análisis que los propios manuales de didáctica formulan. En este apartado, contaré con materiales didácticos de Educación secundaria obligatoria y no obligatoria -Bachillerato-. Para mayor fluidez, a partir de ahora, utilizaré la expresión genérica Educación secundaria para referirme a las dos etapas.

Una constante en las aulas de Educación Secundaria es la distinción categórica entre categorías y funciones, que además se ha respetado a lo largo del marco teórico de este artículo. Así lo especifica claramente el libro de texto de Santillana de $1^{\circ}$ de Bachillerato, al que a partir de ahora me referiré con la referencia (VV. AA., 2008b) y cuya información bibliográfica completa se puede encontrar en la entrada bibliográfica correspondiente a la misma. En este manual se proporciona a los estudiantes una guía de fases del análisis sintáctico (p. 13) cuyas fases 2 y 3 establecen la categorización en cuestión:

4) 2. Determinación de la clase a la que pertenece la unidad (...)

3. Descripción de las relaciones sintácticas que mantiene la unidad con otras unidades (determinación de la función).

Así en El tren llega con retraso, el tren (...) es un SN formado por un núcleo nominal - tren-y un artículo -él-(forma).

Por lo tanto, desde el comienzo, se insta a los estudiantes a establecer dos planos diferenciados: el de las categorías gramaticales (la forma) y el de las funciones sintácticas. Como se observa en la cita 4, además, en el plano de la forma, se refiere directamente a los artículos. Sin embargo, a la hora de desglosar las clases gramaticales y sus funciones, este libro de texto no resulta claro, como esperaríamos de un material didáctico. En el epígrafe dedicado al SN y sus constituyentes (VV. AA., 2008b, p. 88), se introduce el término determinante, opuesto a complemento del nombre, para referirse al tipo de constituyente del lado izquierdo del nombre. Efectivamente, se reitera la idea de que los artículos (y lo demostrativos, posesivos, cuantificadores...) son las "clases de palabras" que pueden ostentar la etiqueta de determinante; no obstante, en el ejemplo propuesto (reproducido parcialmente en la etiqueta determinante aparece en el "plano de la forma" y entre paréntesis (lo que parece indicar que redunda), y lo que encontramos en el "plano de la función” es la etiqueta especificador (p. 88).

5)

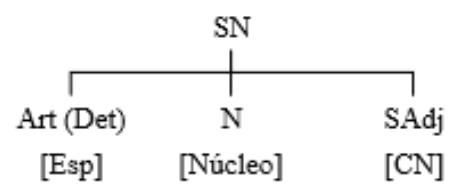


En la línea de los primeros análisis de corte generativo, en este libro de texto se propone la función de especificador del núcleo a los complementos "con significado gramatical” que aparecen al lado izquierdo (VV. AA., 2008b, p. 14). Sin embargo, no queda claro, en primer lugar, en qué se diferencian las etiquetas determinante y especificador, puesto que en unos casos es la primera la que se opone a complemento (p. 88), mientras que en otros es la segunda (p. 14); y, en segundo lugar, en qué se diferencia la etiqueta de determinante de las que aluden a clases de palabras (artículo, demostrativo, numeral, etc.). En unos casos, parece que se adopta la perspectiva que veíamos en Fernández Leborans (1983) (p. 88); y, en otros, parece que se adopta la perspectiva de la teoría X-con-barra, clasificando bajo la función de especificador a los determinantes y adverbios a la izquierda de nombres y adjetivos respectivamente (p. 14). No podemos dejar de notar el hecho de que en este último caso se incurre en una incoherencia al poner al mismo nivel los determinantes, que no se han considerado clases gramaticales, y los adverbios.

La misma editorial, en el manual de $1^{\circ}$ de ESO, al que a partir de ahora me referiré con la referencia VV. AA. (2009) se opta por una clasificación diferente, aunque no mucho más transparente. Si bien en un primer momento aportan una clasificación en torno a las clases de palabras de corte clásico que, en el terreno de lo nominal, solo distingue entre sustantivos y adjetivos calificativos (p. 28), a lo largo del desarrollo del temario de lengua, se incluyen dos epígrafes dedicados, al artículo, por un lado, al que se le atribuye la función sintáctica de determinante; y a los adjetivos determinativos, por otro, a los que en un primer momento se presenta como un subtipo de la clase gramatical de los adjetivos (p. 150), pero después se los define como una variante de los determinantes (p. 164):

6) Normalmente, los sustantivos comunes van precedidos de un determinante (...) Además del artículo (...), pueden desempeñar esta función los adjetivos determinativos (...)[:] posesivos, demostrativos, indefinidos, numerales, interrogativos y exclamativos.

En este marco, resulta confuso qué conceptos denotan clase, qué conceptos función: si los adjetivos calificativos son una clase gramatical en sí misma, ¿lo son también los adjetivos determinativos? Si es así, ¿qué función sintáctica tienen? ¿Qué objeto gramatical son los elementos que componen esa clase? No parece, por lo tanto, que sea posible llegar a un aprendizaje significativo de la gramática a través de este enfoque. Hemos de añadir a esto el hecho de que en los diferentes niveles se manejen clasificaciones que taxonomizan los conceptos de formas y con terminología diferentes.

En los manuales de otras editoriales no se proporcionan materiales más claros: en el libro de 1. ${ }^{\circ}$ de Bachillerato de Oxford educación (Arroyo Cantón y Berlato Rodríguez, 2012) se explica que determinantes son un tipo de complemento del núcleo nominal y que esa función - no categoría gramatical- la pueden desempeñar artículos, adjetivos demostrativos, posesivos, etc. (p. 71); sin embargo, en el apartado correspondiente a las categorías o clases de palabras no aparece ninguna de las mencionadas. En el libro de 
texto de Edelvives para 3. ${ }^{\circ}$ de ESO (Andrés Ferrer, González Gómez, y Jiménez Escobar, 2010) sí se incluye, en un primer momento, a los determinantes entre las clases de palabras (p. 37) y, para salvar la asimetría forma-función, se aclara que con esa etiqueta se designa tanto la categoría como la función de los artículos y los adjetivos determinativos, incluyendo en esta última denominación y a los problemáticos demostrativos, posesivos, etc. (p. 40). ${ }^{11}$ Esta opción no resulta menos confusa, puesto que, aunque lo haga explícitamente, sigue mezclando dos tipos de etiquetado (el formal y el categorial). Esta editorial defiende el mismo análisis ilustrado en 5. Este tipo de análisis tan extendido crea una asimetría en el sintagma. Observamos cómo se defiende el etiquetado de los complementos del lado derecho como sintagma - con la categoría que le corresponda- en el plano de la forma, mientras que sistemáticamente etiqueta en forma de categoría los complementos del lado izquierdo: artículo, determinante, adverbio... Como ya hemos visto, contar con dos expresiones mínimas en un sintagma conforma una gran incoherencia gramatical.

El caso del libro de texto de Akal (VV. AA., 2008a) es diferente, puesto que desde el comienzo se aclara la problemática en torno a las categorías de determinante y pronombre (p. 31). Con todo ello, se establece una distinción clara y categórica entre el plano de la forma y el de la función: determinantes y pronombres son categorías gramaticales y, por lo tanto, pertenecen al análisis de la forma; en el plano de la función, los autores de este manual optan por la etiqueta de modificador para los determinantes y aclaran que los pronombres cumplen la función de núcleo (p. 32). También proponen una solución para esas "categorías problemáticas": demostrativos, posesivos, indefinidos, numerales, relativos, interrogativos, exclamativos y pronombres personales son una subclase de determinantes y pronombres (p. 31) ${ }^{12}$ Esta modelo de análisis es la misma que propone Gómez Torrego (2010: cap. 2.1), cambiando únicamente la etiqueta de modificador por la de actualizador $^{13}$.

Como hemos visto a lo largo de este apartado, no es fácil encontrar materiales didácticos que expongan de manera clara la estructura del SN y propongan un modelo de análisis coherente con sus propias descripciones gramaticales. Aunque hemos encontrado un manual en el que sí había una propuesta firme (VV. AA., 2008a), sigue teniendo claros matices tradicionales que entran en contradicción con lo que muestran hoy los estudios de lingüística. Adoptar una perspectiva basada en modelos de análisis actualizados será lo que nos permitirá salvar algunos problemas con los que se topó la gramática tradicional.

11 Me parece relevante que esta editorial hace notar la habitual confluencia de determinantes y pronombres.

12 Como se desprende de ello, mantienen la distinción tradicional entre adjetivos determinativos y pronombres, aunque de forma actualizada.

13 Actualizador, sin embargo, es una denominación que presenta bastantes problemas, como explican Eguren y Fer nández Soriano (2006: 21-22). 


\section{PROPUESTA DE ACTUALIZACIÓN METODOLÓGICA}

A continuación, procedo a exponer la propuesta metodológica del trabajo. Dado que los contenidos tratados no corresponden si quiera a una unidad completa del currículo de Educación secundaria, no considero apropiado formalizarlo en forma de secuenciación didáctica tradicional, puesto que los contenidos deberán quedar enmarcados en una unidad didáctica mayor. Se expondrán únicamente los contenidos en forma de epígrafe y las orientaciones metodológicas correspondientes a cada sección. Los contenidos descritos en esta sección se adscriben al tema del SN de cada nivel educativo y los objetivos, criterios y estándares de evaluación son los correspondientes a tal unidad. La secuencia queda evidenciada en la estructura de epígrafes de este apartado: en primer lugar, definir qué es un determinante y qué clase de elementos forman parte de esta categoría; en segundo lugar, explicitar la diferencia entre SN y SD y justificar la necesidad de esta distinción; en tercer lugar, estructurar el repertorio de expresiones que forman la categoría de los determinantes; por último, estudiar la estructura sintáctica de los SSDD y sus posibles manifestaciones.

\section{1. ¿Qué es un determinante?}

Para comenzar, es importante conocer qué es un determinante y qué tipo de palabras forman ese repertorio. Habrá que comenzar explicando que la gramática se estructura en torno a una serie de clases de palabras o categorías. Habitualmente nos referimos a las categorías léxicas (sustantivos, adjetivos, verbos y adverbios); sin embargo, hay otras categorías de tipo gramatical entre las que se encuentra la de los determinantes $-\mathrm{y}$ la de los pronombres-. Para una caracterización detallada de los determinantes como categoría gramatical se puede recurrir a los libros de texto Andrés Ferrer et al. (2010) y VV. AA. (2008a).

Es importante aclarar que las tradicionales clases de artículo, demostrativo, posesivo, etc. actualmente no se incluyen entre las categorías gramaticales, puesto que no hay muestras de que sean visibles a la sintaxis de la oración. Por lo tanto, su único interés gramatical reside en los paradigmas propios que configuran y en los matices de significado que aportan. Todas ellas son en realidad subtipos de determinantes, como veremos a continuación.

\subsubsection{Los artículos}

Son el determinante que tradicionalmente se considera central. Esto se debe a que es el único determinante cuya única función es referencial: no añade ningún tipo de significado conceptual. Únicamente nos sirve para reconocer que nos referimos a una expresión ya conocida por nuestro interlocutor. 
Para la descripción de las propiedades semánticas del artículo se puede recurrir a cualquier libro de texto o manual didáctico de Lengua española ${ }^{14}$. Se recomienda, además, mostrar a los estudiantes el paradigma de los artículos del español para que sean capaces de identificar correctamente sus formas a la hora de realizar los análisis.

En los niveles más avanzados, se puede introducir la noción de determinación fuerte y determinación débil, como explica Fernández Leborans (2003: 39), ya que esta distinción podría facilitar el análisis y reconocimiento de los sujetos posverbales. Recordemos que estos solo pueden ser SSNN o SSDD débiles (Hay unos hombres/*Hay los hombres, Fueron encontradas unas joyas $/{ }^{*}$ Fueron encontradas las joyas).

\subsubsection{Los "adjetivos determinativos"}

En este punto es clave tratar el asunto de los tradicionales adjetivos determinativos, puesto que, en muchos casos, los estudiantes habrán estado en contacto previo con esta terminología. Se debe aclarar el error de incluir a los posesivos, demostrativos, numerales e indefinidos en la categoría de los adjetivos aun añadiéndole la etiqueta de determinativos, puesto que, como señalan Eguren y Fernández Soriano (2006: 16), si lo fueran no existirían los contrastes siguientes, donde se compara la sustitución de uno de estos elementos por adjetivos:

7) a. Esta novela ha recibido un premio./*Sorprendente novela ha recibido un premio.

b. Cada actor representó un papel en la obra./*Antiguo actor representó un papel en la obra.

c. Muchos profesores suspenden a pocos alumnos. $/{ }^{*}$ Verdaderos profesores suspenden a pocos alumnos.

Si estas palabras fuesen adjetivos, no habría agramaticalidad al sustituirlas por otros adjetivos; sin embargo, lo cierto es que no lo son. Son una clase de palabra diferente: determinantes. Por ello, sí que resulta posible intercambiarlos con artículos: La novela..., El actor..., Los profesores... Por lo tanto, hemos de considerar a todas estos elementos una subclase de determinante, que, a su vez, forma una categoría gramatical como los sustantivos, los adjetivos, los verbos, etc.

\subsection{Sintagma nominal y sintagma determinante}

En esta sección se explicarán las diferencias clave entre los dos tipos de estructura nominal. El SN es el tipo de sintagma que tiene como núcleo de significado al nombre. Como ya sabemos, los nombres o sustantivos son un tipo de palabras que nos evocan imágenes

\footnotetext{
14 Recordemos que la descripción de los elementos gramaticales no era el problema de estos recursos didácticos, sino la forma de clasificación y la coherencia a lo largo de las unidades.
} 
de los elementos que existen en el mundo real. En este punto es importante aclarar que los nombres no sirven para "referirse" a objetos, sino que su función es evocar o traer a nuestra mente conceptos del mundo real. Si decimos ventana, esa palabra -por sí solano tiene suficiente fuerza para hablarnos de ningún objeto concreto del mundo real: no se puede decir que ventana exista ni represente ningún objeto del mundo. Debemos añadirle otro elemento delante: el determinante. Desde el momento en que decimos $\{\mathrm{la} / \mathrm{esa} /$ su/una/etc. $\}$ ventana, hacemos que la ventana exista, que sea una ventana del mundo real. El determinante es fundamental, además, porque es el que determina lo que vamos a entender finalmente. No es lo mismo la ventana, su ventana, esa ventana, otra ventana, etc. Como esto es así: decimos que, aunque el núcleo de significado - el que aporta el contenido descriptivo- es el nombre, el núcleo de la estructura sintáctica es el determinante, puesto que es el que decidirá dónde y cómo podremos insertar ese sintagma.

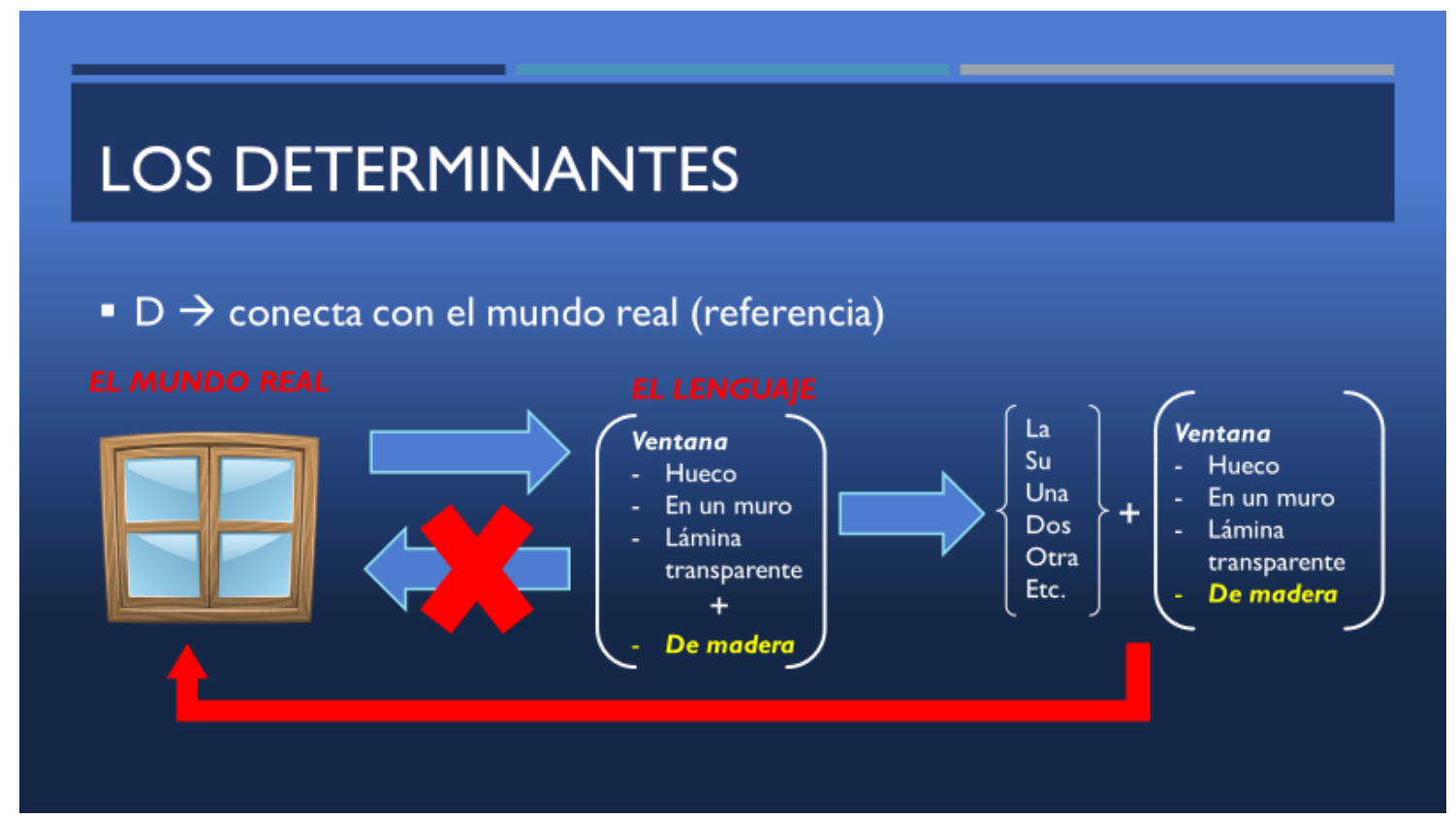

Ilustración 1: Ejemplo de material de apoyo para el desarrollo del epígrafe 4.1. Se muestra la importancia del determinante a la hora de obtener la referencia de un nombre.

La explicación se puede acompañar de apoyo audiovisual o de alguna diapositiva que ilustre los contenidos. La ilustración 1 ofrece un ejemplo. En ella se muestran dos planos: el mundo real -el de los objetos- y el lenguaje -el de las palabras-. En el mundo del lenguaje hay dos zonas diferenciadas: una primera columna, con una matriz de significados que representa al SN, y una segunda zona en la se añade el determinante a esa matriz. Se observa como los nombres encarnan ideas que proceden del mundo real (la flecha que conecta la ventana con la matriz); sin embargo, esos nombres no pueden conectar por sí solos con elementos concretos del mundo real (la flecha con una cruz roja). Dentro de la matriz de significados hay una expresión en amarillo que representa el complemen- 
to del nombre ${ }^{15}$. Incluso con complementos del nombre que especifiquen el significado del núcleo, el SN no tiene la capacidad de referirse a objetos específicos.

Únicamente conseguimos conectar la expresión nominal con el objeto al que queremos referirnos cuando añadimos el determinante, que es el que determinará finalmente el significado del sintagma. SN y SD, por lo tanto, son dos objetos con funciones diferentes y, como veremos en el siguiente epígrafe, estructuras diferentes.

\section{3. ¿Por qué un SD?}

Podríamos pensar en por qué es necesario hablar de una categoría sintáctica nueva si hasta ahora analizábamos esas mismas estructuras bajo una misma etiqueta. Pues bien, en lengua, como en todas las ciencias, se introduce un nuevo concepto cuando existen una serie de procesos que afectan únicamente a un grupo concreto de elementos. Cuando esto ocurre, se dice que estos elementos forman un grupo o clase diferenciado del resto. En biología, se habla de mamíferos porque este grupo de seres vivos tienen unas determinadas características propias que los diferencian del resto y hay una serie de procesos naturales que los afectan de forma separada al resto de seres. Si algo fuera igual que el res to, no tendría sentido llamarlo de otra forma ni tratarlo por separado. Los SSDD cumplen estas condiciones:

- Les afecta la selección sintáctica, ya que en posición preverbal los sujetos solo pueden ser un SD.

- Les afecta la selección semántica: por ejemplo, cuando en una posición podemos elegir entre un SN o un SD, la elección de uno u otro condiciona el significado de la oración (ver ejemplos en 3).

- De igual modo, tienen unas condiciones estructurales fijas: un determinante siempre selecciona como complemento otro SD o un SN.

\subsection{La estructura del SD}

La última edición de la gramática de la RAE (RAE y ASALE, 2009) ya incluye la noción de que el determinante afecta a la interpretación final del sintagma y que los sintagmas no conforman estructuras lineales, sino jerárquicas. De este modo, el determinante afecta a la interpretación del conjunto nombre + complemento del nombre y no únicamente al nombre ${ }^{16}$. La estructura tradicional en cajones no nos permite dar cuenta de los elemen-

15 Esto representa gráficamente la idea señalada en el marco teórico de que los complementos del lado derecho del sintagma nominal añaden propiedades intensionales o descriptivas; es decir, no permiten obtener referencia, simplemente restringen el significado.

16 En esta línea se mostraba la ilustración 1, donde las nociones de los complementos del nombre (color amarillo) se incluían en la matriz del sintagma nominal y al conjunto de todo ello se añadía el determinante. 
tos más altos (más fuertes, dominantes) y los más bajos (dominados). Por esto, se recomienda utilizar la estructura en árbol, como en los ejemplos de 8.

Como el determinante es el constituyente más alto, afecta a todo el SN. Y, de esta for ma, por primera vez, podemos dar cuenta de la importancia de los determinantes. El SD y el SN son dos sintagmas ensamblados pero autónomos: cada uno tiene su estructura interna independiente con sendos núcleos, complementos y especificadores. La posición de especificador es esencial, puesto que sin ella no podríamos explicar estructuras como las de 8, donde a. incluye un especificador del SD, y b. uno del SN.

8) a.

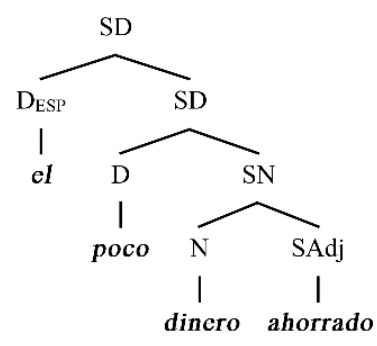

b.

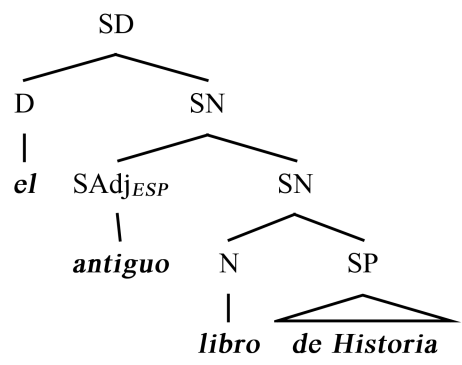

\subsubsection{El SN sin nombre}

Aunque este epígrafe debería formar parte del apartado concreto dedicado al SN, es importante resaltar la existencia de estructuras nominales sin el nombre. Tradicionalmente, estas estructuras se denominaban sustantivadas; sin embargo, como demuestran Eguren y Fernández Soriano (2006: cap. 1.3), no es así. En estructuras como Quiero las azules, debemos reconocer que hay un nombre omitido, puesto que no es posible entender esa secuencia sin que previamente hayamos mencionado a qué entidades azules nos referimos. El análisis de estas secuencias, por tanto, debe incluir un SN, cuyo núcleo está omitido, mediando entre el SD y el sintagma adjetival (complemento del nombre).

\subsubsection{El SD sin determinante}

Este epígrafe está concebido para introducir, en primer lugar, los pronombres que, como veíamos en el marco teórico, forman SSDD y no SSNN. De esta forma, hay que explicar 
a los estudiantes la proximidad entre las categorías de determinante y de pronombre en cuanto a sus propiedades referenciales y que ambas se proyectan en SSDD. Por lo tanto, en este caso, estaríamos ante sintagmas determinante sin un determinante. Sería óptimo hacer notar que estas eventualidades no son inusuales: en el apartado anterior hemos visto que es posible formar SSNN sin el nombre núcleo.

\section{CONSIDERACIONES FINALES}

Tras todo el trabajo, podemos concluir que la enseñanza de las estructuras nominales a través de los materiales didácticos tradicionales está, en la mayoría de los casos, condenada a caer en el error de no distinguir entre dos categorías fundamentales: el nombre y el determinante. La confusión de sus funciones y de sus estructuras lleva a contradicciones internas a la gramática y a incoherencias en el discurso didáctico.

Asimismo, se ha podido observar que las nociones más actualizadas de gramática no están reñidas con la enseñanza de la lengua en Educación secundaria, puesto que todo contenido es susceptible de adaptarse al nivel necesario. En esta propuesta de actualización metodológica, hemos visto cómo es posible adaptar las cuestiones en torno al sintagma determinante a los objetivos gramaticales básicos de la enseñanza. Esto, además, nos permite romper con la linealidad y monotonía de los contenidos del área de lengua y poder ampliar progresivamente los conceptos hasta alcanzar los más complejos, como los diferentes tipos de determinante o las estructuras nominales complejas.

Es importante que los docentes de Educación secundaria no solo cumplan con los contenidos y objetivos esenciales del currículo, sino que, además, doten a los estudiantes de un conocimiento actualizado y con mayor coherencia.

\section{REFERENCIAS}

ABNEY, STEVEN P. (1987): The English noun phrase in its sentential aspect (Tesis doctoral), M.I.T., Massachussets.

ALARCOS LLORACH, EMILIO (1951): Gramática estructural, Madrid, Gredos.

ANDRÉS FERRER, PALOMA, M. GONZÁLEZ GÓMEZ, Y M. JIMÉNEZ ESCOBAR (2010): Lengua castellana y Literatura (Vol. 1), Zaragoza, Edelvives.

ARROYO CANTÓN, CARLOS, Y P. BERLATO RODRÍGUEZ (2012): Lengua castellana y Literatura, Madrid, Oxford Educación.

BELLO, ANDRÉS (2002): Gramática de la lengua castellana destinada al uso de los americanos, Alicante, Biblioteca Virtual Miguel de Cervantes.

BOSQUE, IGNACIO (2007): Las categorías gramáticales. Relaciones y diferencias, Madrid, Síntesis. 
BOSQUE, IGNACIO, Y J. GUTIÉRREZ-REXACH (2009): Fundamentos de sintaxis formal, Madrid, Akal.

BRUCART, JOSÉ M., Y M. L. HERNANZ (2015): "Las posiciones sintácticas", en Perspectivas de sintaxis formal, GALLEGO, Á. J. (ed.), Madrid, Akal, 33-104.

CHOMSKY, NOAM A. (1970): "Remarks on nominalizations", en Readings in English Transformational Grammar, JACOBS, R. A. Y P. S. ROSENBAUM (eds.), Waltham, Ginn, 184-221.

EGUREN, LUIS J., Y O. FERNÁNDEZ SORIANO (2004): Introducción a una sintaxis minimista. Madrid, Gredos.

EGUREN, LUIS J., Y O. FERNÁNDEZ SORIANO (2006): La terminología gramatical. Madrid, Gredos.

ESCANDELL VIDAL, MARÍA V. (2004): Fundamentos de Semántica composicional. Barcelona, Ariel.

FERNÁNDEZ LEBORANS, MARÍA J. (1983): "Notas sobre el sintagma en la lengua española”, DICENDA - Cuadernos de filología hispánica, 2, 57-75.

FERNÁNDEZ LEBORANS, MARÍA J. (2003): Los sintagmas del español (Vol. I, El sintagma nominal), Madrid, Arco Libros.

GÓMEZ TORREGO, LEONARDO (2010): Gramática didáctica del español, Madrid, SM.

JACKENDOFF, RAY S. (1977): The Architecture of the Language Faculty, Cambridge, Mass, The MIT Press.

RAE (1931): Gramática de la lengua española, Madrid, Espasa Calpe.

RAE, y ASALE (2009): Nueva gramática de la lengua española. Morfología y Sintaxis I (Vol. 1), Madrid, Espasa.

VV. AA. (2008a): Lengua castellana y Literatura 1.o Bachillerato (J. Rodríguez Puértolas, ed..), Madrid, Akal.

VV. AA. (2008b): Lengua y Literatura para 1.o de Bachillerato (P. López Lara, ed.), Madrid, Santillana.

VV. AA. (2009): Lengua y Literatura para 1o de ESO. (F. López Martínez, ed.), Madrid, Santillana. 